\title{
Hydrogel coated mesh decreases tissue reaction resulting from polypropylene mesh implant: implication in hernia repair
}

\author{
D. P. Poppas ${ }^{1}$ J. J. Sung ${ }^{2}$ C. M. Magro' ${ }^{2}$ J. Chen ${ }^{1}$ J. P. Toyohara' ${ }^{2}$ \\ B. J. Ramshaw ${ }^{3}$ D. Felsen ${ }^{1}$
}

Received: 23 July 2015/Accepted: 4 March 2016/Published online: 21 March 2016

(c) The Author(s) 2016. This article is published with open access at Springerlink.com

\begin{abstract}
Purpose Use of polypropylene mesh (PPM) in hernia repair is associated with tissue reactivity. We examined, in a rat model, a novel non-biodegradable hydrogel coated PPM which may allow for decreased inflammation and a decreased foreign body reaction.

Methods Through a dorsal midline incision, a $2 \mathrm{~cm} \times 2 \mathrm{~cm}$ section of PPM (either coated or uncoated) was placed on the fascial surface $1.5 \mathrm{~cm}$ from the incision on the dorsal wall of Sprague-Dawley rats. At 2 and 12 weeks after placement, the PPM and surrounding tissue were harvested. A board-certified dermatopathologist examined H\&E stained slides for fibrosis and foreign body reaction. In addition, tissues were stained for apoptotic cells, oxidative damage, macrophages, fibroblasts, neovascularization and metalloproteases.

Results At 2 and 12 weeks, there was a greater than $95 \%$ decrease in foreign body giant cells in coated PPM samples compared to uncoated; fibrosis was decreased by $50 \%$. At 2 and 12 weeks, oxidative damage, fibroblast accumulation, apoptosis and macrophages were significantly decreased in coated PPM samples compared to uncoated PPM.
\end{abstract}

D. Felsen

dfelsen@med.cornell.edu

1 Department of Urology, Institute for Pediatric Urology, Weill Cornell Medical College, Box 94, 1300 York Avenue, New York, NY 10065, USA

2 Department of Pathology, Weill Cornell Medical College, New York, NY, USA

3 Department of General Surgery, University of Missouri School of Medicine, Columbia, MO, USA
Conclusion These results demonstrate that a nonbiodegradable hydrogel coating of PPM led to significant reduction in foreign body reaction, oxidative stress and apoptosis compared to uncoated PPM in vivo, and suggest that this coating could be clinically useful in hernia repair.

Keywords Hydrogel - Hernia repair - Polypropylene mesh · Foreign body reaction

\section{Introduction}

Since the introduction of Marlex mesh by Usher in 1958 [1], polypropylene mesh (PPM) has become a mainstay in hernia repair [2,3]. While it has revolutionized the field, PPM has also been associated with significant morbidities including, pain, erosion, migration and adhesion formation [4-6]. Since both surgical techniques and indications for mesh placement vary, development of an "ideal" mesh, suitable for all purposes, may not be possible. However, it is generally agreed that limiting the foreign body response (FBR) is an important step in developing a biocompatible mesh.

The FBR is part of the cascade of events that occurs when a foreign material is implanted. Occurring to different degrees in different tissues, the response to implanted materials includes blood-material interactions, acute inflammation, chronic inflammation, FBR, and fibrous encapsulation [7]. Briefly, when a biomaterial is implanted, it is immediately coated with surface proteins. At the same time, acute inflammation occurs, as neutrophils are attracted to the implanted biomaterial. The neutrophils attempt to engulf the biomaterial, but its large size prevents this, and leads to a process known as "frustrated phagocytosis" [8] and release of inflammatory mediators at the 
site. This is followed by entry of other inflammatory cells, including monocytes, macrophages and lymphocytes, along with neovascularization and proliferation of connective tissue, resulting in a chronic inflammatory process. Chronic inflammation is followed by the FBR, which is the accumulation of both foreign body giant cells (FBGC), which are fused macrophages, and granulation tissue, the aggregation of new blood vessels and connective tissue. Finally, there is a fibrotic encapsulation of the biomaterial which follows the FBR.

In addition to limiting the FBR, an ideal mesh would be one that was "technically easy to place, did not incite a chronic inflammatory response, did not degrade over time, resulted in minimal shrinkage, was resistant to infection, resulted in excellent healthy tissue integration, and no adhesion formation, small-bowel obstructions, or fistulas, and resulted in a durable repair" [9]. In this paper, the properties of a non-biodegradable hydrogel coated PPM are demonstrated in comparison to a bare uncoated PPM. A hydrogel coating for PPM has been developed which has a novel Polyurethane backbone, similar to polyurethanes that have been used for long-term implants in patients (see "Methods"). In this study, we tested the hydrogel coated PPM and compared it to an uncoated PPM to determine the potential of the hydrogel coated PPM to address the limitations of the naked PPM that are commercially available, by lessening the inflammatory and foreign body reaction in an animal model.

\section{Methods}

\section{Hydrogel coating}

The non-biodegradable hydrogel coating is a synthetic, long chain polyol on a Polyurethane backbone, and was supplied by promethean surgical devices (PSD; East Hartford, CT). A schematic of the polymer, depicting its novel trifunctionality, is shown in Fig. 1. Hydrophilic zones, depicted by blue circles, draw water into the polymer, while hydrophobic zones (green circles) expel water from the polymer. The red circles depict linking groups that provide for crosslinking of the hydrogel on the PPM. The presence of both hydrophilic and hydrophobic sites on the polymer surface allows for the constant exchange of water molecules on the hydrogel surface and its immediate environment. The hydrogel coating used in this study is FDA approved and has passed the following preclearance tests: 10993-3, genotoxicity; "Big Blue" transgenic mouse model, carcinogenicity; 10993-4, hemolysis of blood; 10993-5, cytotoxicity; 10993-6, implantation compatibility; 10993-10, sensitivity; 10993-11, systemic toxicity. PPM was coated in a proprietary process with hydrogel in a commercial facility (PSD). Both light micrographs (A, B) and SEM images $(C, D)$ are shown in Fig. 2. The monofilaments of the mesh, the interstices and the knots are clearly visible in the uncoated mesh, whereas they are covered $(>98 \%)$ in the coated mesh.

\section{PPM implantation}

Sprague-Dawley rats were used for these studies, which were carried out at the University of Missouri School of Medicine. Either coated or uncoated PPM was implanted and animals were euthanized at either 2 weeks ( $n=10 /$ group; acute) or 12 weeks $(n=8 /$ group; long term). To implant the PPM, rats were anesthetized and a $2 \mathrm{~cm}$ longitudinal incision was made off the left dorsal midline. This approach has been determined to prevent animal self-mutilation of wounds when compared to a ventral incision to study implant tissue reactions. The subcutaneous tissues were bluntly dissected off of the central and right-sided abdominal musculature to create a subcutaneous pocket. A $2 \mathrm{~cm} \times 2 \mathrm{~cm}$ piece of sterile PPM was placed on the fascial surface at least $1.5 \mathrm{~cm}$ from the incision; four 4-0 Prolene sutures were used to fix and mark the PPM location. At 2 and 12 weeks, a $3 \mathrm{~cm}$ full thickness section of the dorsal wall was harvested for analysis. All animal protocols were approved by the IACUC and followed institutional guidelines.

\section{Histology}

Tissue samples were embedded in paraffin and sections were made. The histologic analysis was carried out at Weill Cornell Medical College by independent board-certified dermatopathologists, blinded to the treatment. $H \& E$ stained slides were analyzed for fibrosis, foreign body giant cells, eosinophils, polymorphonuclear leukocytes (PMN), histiocytes and lymphocytes.

\section{Immunohistochemistry}

Slides were stained for oxidative stress [staining for 8-hydroxydeoxyguanosine (8-OHdG)], apoptotic cells (TUNEL assay), macrophages and fibroblasts as previously described $[10,11]$. Cells were counted in 10 high power fields (HPF). Neovascularization was identified by staining for CD31 (Santa Cruz, Monoclonal antibody). Staining for matrix metalloprotease-2 was also performed.

\section{Attenuated total reflectance Fourier transform infrared spectroscopy (ATR-FTIR)}

ATR-FTIR was used to measure surface mesh oxidation [12]. ATR-FTIR measures IR intensity versus wavelength 
Fig. 1 Schematic of hydrogel polymer. The hydrogel comprises hydrophilic zones (blue circles) that draw water into the polymer and hydrophobic zones (green circles) that expel water from the polymer. Linking groups (red circles) provide for crosslinking of the hydrogel on to the mesh

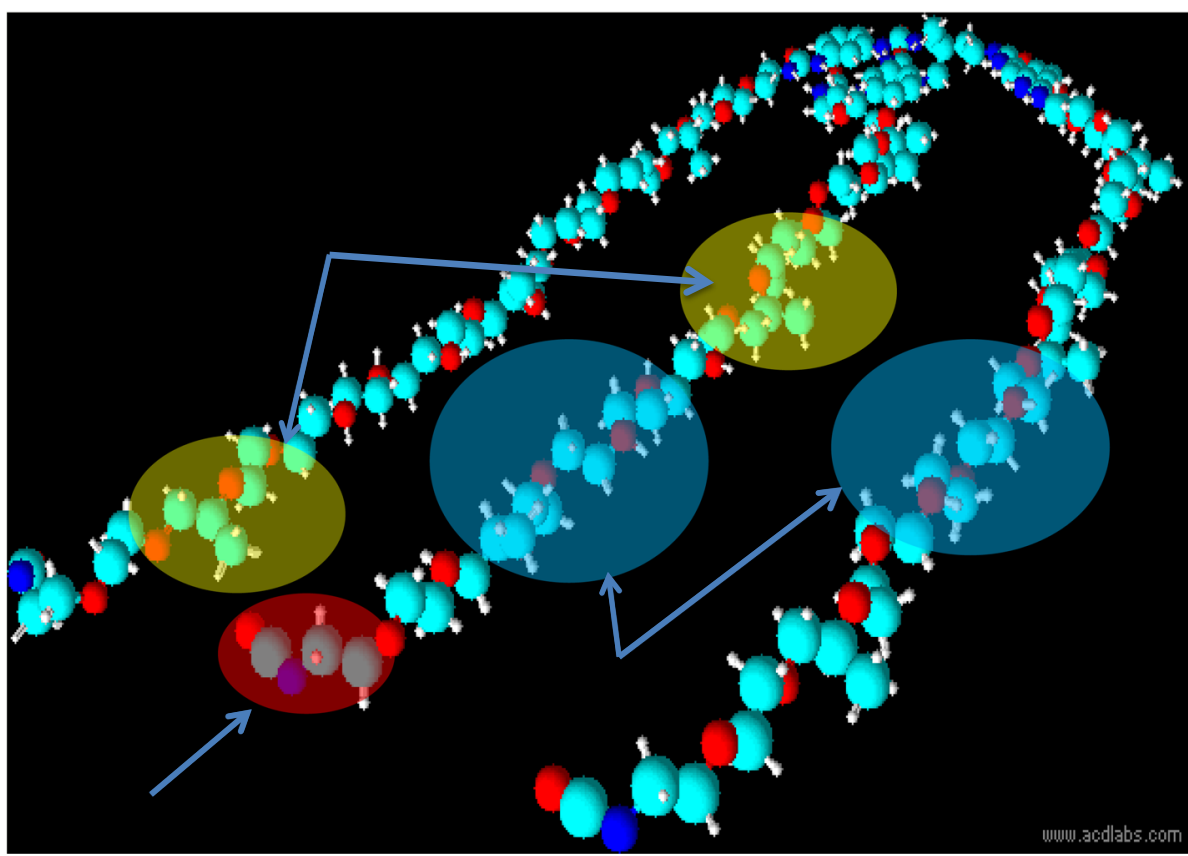

A

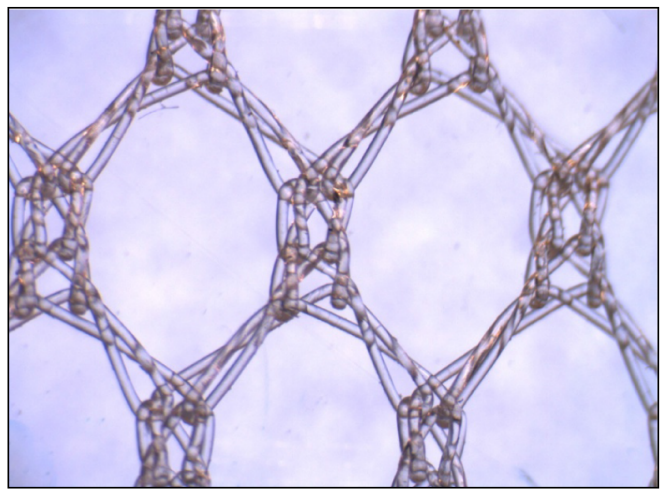

C coated PPM. Light microscope images $(\times 50$ magnification $)$ of uncoated (a) or coated (b) PPM. SEM images $(\times 50$ magnification) of uncoated (c) or coated (d) PPM
B

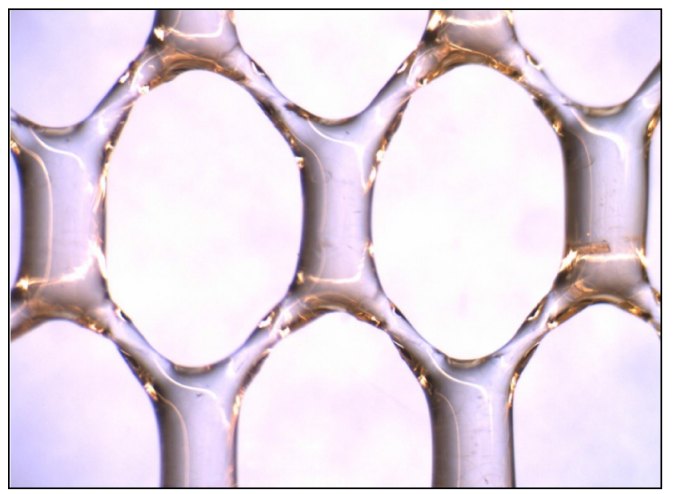

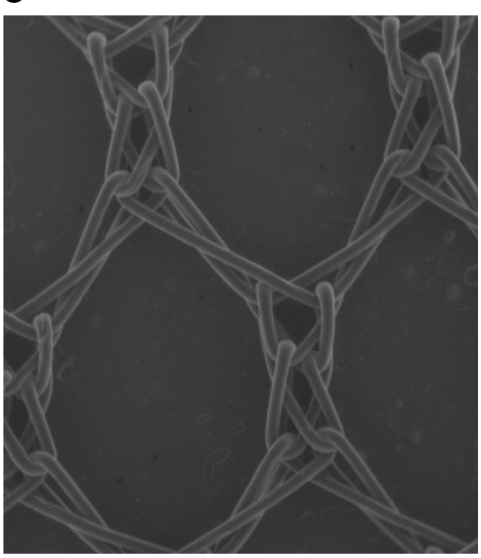

D

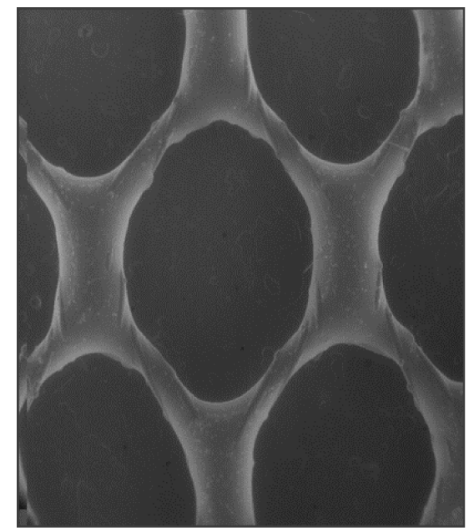

of light by detecting vibration characteristics of chemical functional groups, and detects if chemical changes to the surface of the material are present. Samples of PPM were removed from the rats and placed in sodium hypochlorite for $2 \mathrm{~h}$ (uncoated PPM) or $3 \mathrm{~h}$ (coated PPM; the coating necessitated a longer incubation time, which did not affect 
the results). After cleaning and drying, PPM was placed on the ATR crystal and subjected to ATR-FTIR. The presence of a carbonyl peak was analyzed via a peak area integration program in the OMNIC software package.

\section{Statistical analysis}

All data are reported as mean \pm SEM. We performed the Wilcoxon rank sum test (non-parametric test) to compare differences between groups, with $p<0.05$ considered significant.

\section{Results}

\section{Histology}

At 2 weeks following implantation, the tissue was evaluated for the presence of foreign body giant cells (FBGC), other inflammatory cells and fibrosis. There were $1.78 \pm 0.59 \mathrm{FBGC} / \mathrm{HPF}$ in areas adjacent to uncoated PPM, whereas there were only $0.06 \pm 0.02$ FBGC in areas adjacent to coated PPM $(p<0.01)$ (Table 1; Fig. 3a, b). There were significantly less histiocytes and eosinophils as well in the coated PPM samples (Table 1). The lack of reactivity was confirmed by the finding that there was also a significant decrease in fibrosis in the coated PPM samples as compared to the uncoated $(0.09 \pm 0.03$ vs. $0.18 \pm 0.06 \mathrm{~mm}$, respectively, $p<0.05$; Fig. $3 \mathrm{c}$, d). At 12 weeks, the results were very similar (Table 1). FBGC were still decreased by $90 \%$ in coated PPM, compared to uncoated. Eosinophils and histiocytes were significantly decreased and there was $50 \%$ less fibrosis around the coated PPM. There were significantly more macrophages in the uncoated samples than in the coated samples (Table 1; Fig. 4a, b) at 12 weeks.

\section{Fibroblast expression}

We examined tissue for the presence of fibroblasts by staining for the presence of Fibroblast Specific Protein-1 [13]. At 2 weeks there were $24.4 \pm 2.2 \mathrm{FSP}^{+}$cells/HPF in areas adjacent to uncoated PPM; in contrast there were only $13.3 \pm 5.4 \mathrm{FSP}^{+}$cells/HPF in areas adjacent to coated PPM (Table 1; Fig. 4c, d). Similar results were found at 12 weeks (Table 1).

\section{Apoptosis}

The TUNEL assay was used to determine if there were apoptotic cells in the tissue. At 2 weeks, in samples from the animals implanted with uncoated PPM there were $13.1 \pm 1.1$ apoptotic cells/HPF (Table 2; Fig. 5a-c) This was decreased by almost $90 \%$ in samples from animals implanted with coated PPM $(1.5 \pm 0.2$ cells/HPF; $p<0.001$ ). At 12 weeks, TUNEL positive cells in the uncoated PPM samples were $11.0 \pm 1.1$ cells per HPF, whereas in the coated mesh PPM there were $2.0 \pm 0.4$ $(p<0.001$; Table 2).

\section{Oxidative stress}

Cells were examined for the presence of $8-\mathrm{OH}$ deoxyguanosine $(8-\mathrm{OHdG})$, which detects the presence of oxidative stress on an intracellular level by attaching to DNA damaged by cellular oxidation. In the samples taken from animals implanted with uncoated PPM at 2 weeks there were $20.3 \pm 1.48$-OHdG positive cells/HPF (Table 2). In coated PPM samples, $6.7 \pm 1.2$ cells/HPF $(p<0.001)$ were found, a decrease of over $70 \%$. At 12 weeks there were $8-\mathrm{OHdG}$ positive cells $31.9 \pm 2.3$ per HPF in uncoated PPM samples, compared to $9.3 \pm 1.7$ in coated $(p<0.001$; Fig. 6a, b). ATR-FTIR was used as an
Table 1 Histological analysis of 2 and 12-week tissue

\begin{tabular}{|c|c|c|c|c|}
\hline & $\begin{array}{l}2 \text { weeks } \\
\text { Uncoated }\end{array}$ & $\begin{array}{l}2 \text { weeks } \\
\text { Coated }\end{array}$ & $\begin{array}{l}12 \text { weeks } \\
\text { Uncoated }\end{array}$ & $\begin{array}{l}12 \text { weeks } \\
\text { Coated }\end{array}$ \\
\hline Fibrosis (mm) & $0.18 \pm .06$ & $0.09 \pm 0.03^{*}$ & $0.16 \pm 0.03$ & $0.05 \pm 0.02 * *$ \\
\hline Foreign Body Giant Cells & $1.78 \pm 0.59$ & $0.06 \pm 0.02 * *$ & $2.11 \pm 0.12$ & $0.20 \pm 0.22^{\dagger}$ \\
\hline Eosinophils & $2.89 \pm 0.96$ & $0.06 \pm 0.06^{\dagger}$ & $1.89 \pm 0.28$ & $0.20 \pm 0.20^{\bullet}$ \\
\hline PMNs & $0.78 \pm 0.26$ & $0.39 \pm 0.14$ & $0.78 \pm 0.16$ & $0.80 \pm 0.22$ \\
\hline Histiocytes & $2.38 \pm 0.79$ & $1.11 \pm 0.39^{\curvearrowleft}$ & $2.56 \pm 0.19$ & $1.60 \pm 0.27 * *$ \\
\hline Lymphocytes & $1.75 \pm 0.58$ & $2.00 \pm 0.71$ & $1.56 \pm 0.19$ & $1.8 \pm 0.22$ \\
\hline Macrophages & $73.3 \pm 11.4$ & $21.8 \pm 2.1^{* *}$ & $42.1 \pm 12.3$ & $22.8 \pm 5.5$ \\
\hline Fibroblasts $\left(\mathrm{FSP}^{+}\right.$cells $)$ & $24.4 \pm 2.2$ & $13.3 \pm 5.4^{\top}$ & $23.5 \pm 2.8$ & $14.8 \pm 2.7^{*}$ \\
\hline
\end{tabular}

Fibrosis (mm) and Foreign Body Giant Cell, Eosinophil, PMN, Histiocyte and Lymphocyte counts were determined by the dermatopathologist. Macrophage and Fibroblast counts were obtained from immunohistochemistry

Compared to Uncoated: $* p<0.05 ; * * p<0.01{ }^{\bullet}{ }^{\circledR} p<0.001 ;^{\dagger} p<0.0001$ 

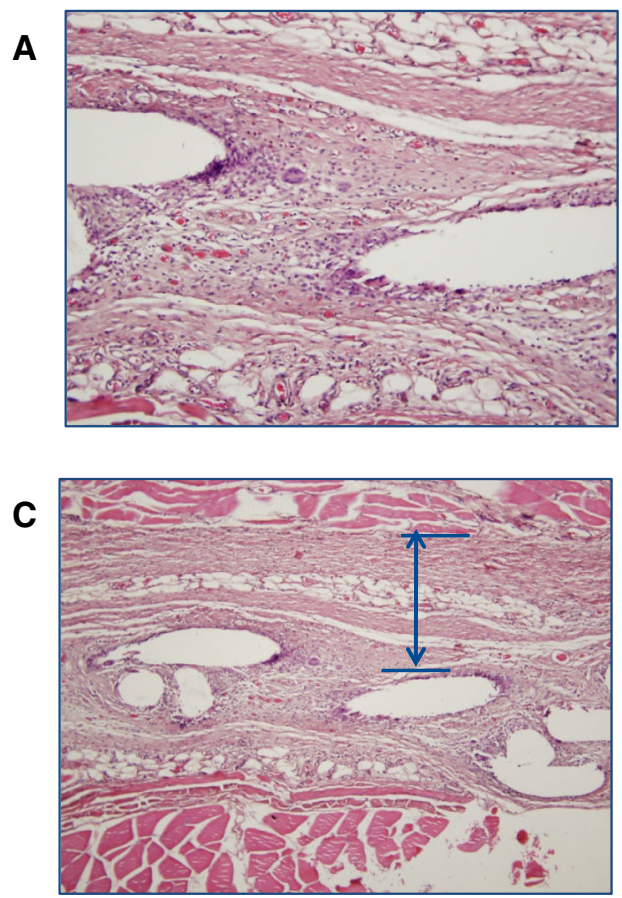

Uncoated PPM

Fig. $3 \mathrm{H} \& \mathrm{E}$ stain of 2-week samples of implanted PPM. H\&E stained samples of both uncoated (a) and coated (b) PPM are shown. In $\mathbf{c}$ (uncoated) and $\mathbf{d}$ (coated) the width of the fibrotic area is shown

indirect measure of the surface oxidation of the mesh. A spectrum is shown in Fig. 7a and a graphical representation of the results is shown in Fig. 7b. The uncoated PPM showed significantly higher carbonyl peaks than the coated PPM $(0.24 \pm 0.22$ vs. $0.07 \pm 0.07 ; p<0.05)$.

\section{Neovascularization}

An antibody to CD31 was used to determine if there were new blood vessels in the samples. We then calculated the CD31-positive area as a percent of the total area. We found that at 2 weeks, the percent $\mathrm{CD}-31$ positive area in the uncoated samples was $9.01 \pm 1.47 \%$ (Fig. 8a, c; Table 2) compared to $14.68 \pm 1.38 ; p<0.01$ in the coated samples (Fig. 8b, d; Table 2). Thus in contrast to measures of tissue damage, which were all less in the coated samples, there was more neovascularization in the coated samples. This is a positive indicator of healthy tissue growth. This difference persisted at 12 weeks.

MMP-2: Tissues were stained for matrix metalloprotease-2. MMP-2 staining in the uncoated PPM was found directly around the implant. In the coated mesh samples there was almost no MMP-2 staining (Fig. 9a, b). This finding supports a lower tissue reaction to coated mesh.

\section{B}

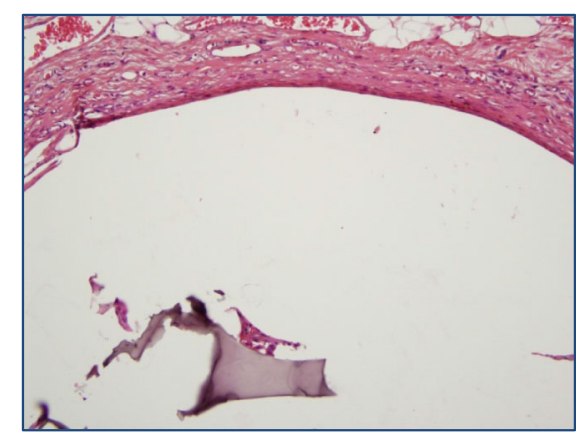

D

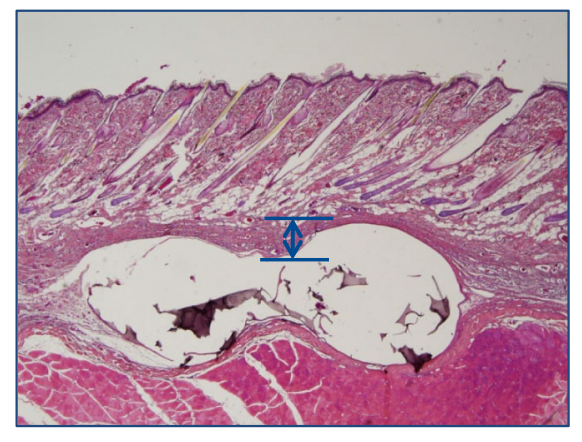

Coated PPM

by the blue arrows. Note the lack of Foreign Body Giant Cells in the coated PPM compared to uncoated. Quantification of fibrosis and Foreign body giant cells is given in Table 1

\section{Discussion}

Polypropylene mesh has become a mainstay of hernia repair $[2,3]$. The strength and durability of the material has improved outcomes, but the reaction to the mesh by host tissues is unpredictable and often undesirable. The desire to minimize inflammatory reactions, while maintaining the integrity of the mesh, has led to the development of materials and changes in geometry (pore size and material weight) in an attempt to preserve the good qualities and mitigate the bad. In this study, we demonstrated that coating PPM with a novel non-biodegradable hydrogel resulted in a significant decrease in the immediate and chronic foreign body responses. The number of foreign body giant cells found at 2 weeks was decreased by $95 \%$ and this persisted over 12 weeks. Fibrotic encapsulation was greatly decreased, as were apoptotic cells, oxidative stress and MMP-2 expression. Thus, coating PPM with hydrogel can significantly mitigate the negative reactions associated with uncoated PPM in use today for surgical repair of hernias.

Curtailing the FBR should mitigate many of the negative reactions of the host to PPM implantation. The first stage of the acute FBR is the adsorption of proteins onto the surface of the implant. Once proteins adsorb, they can be replaced 
A

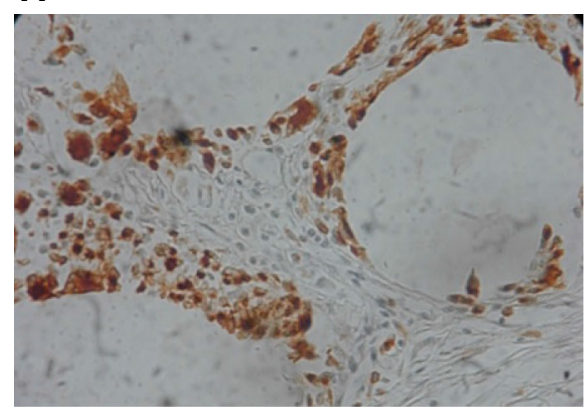

C

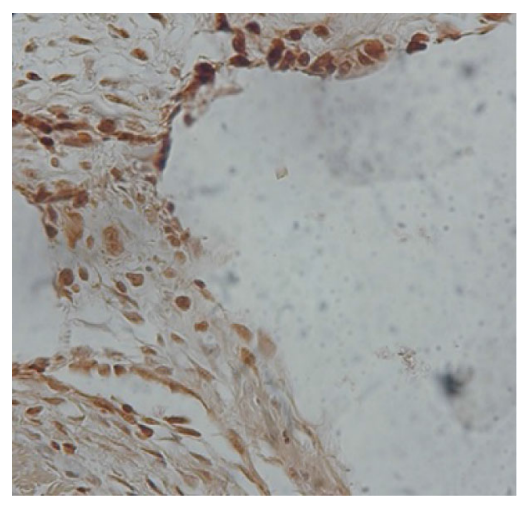

Uncoated PPM

D
B
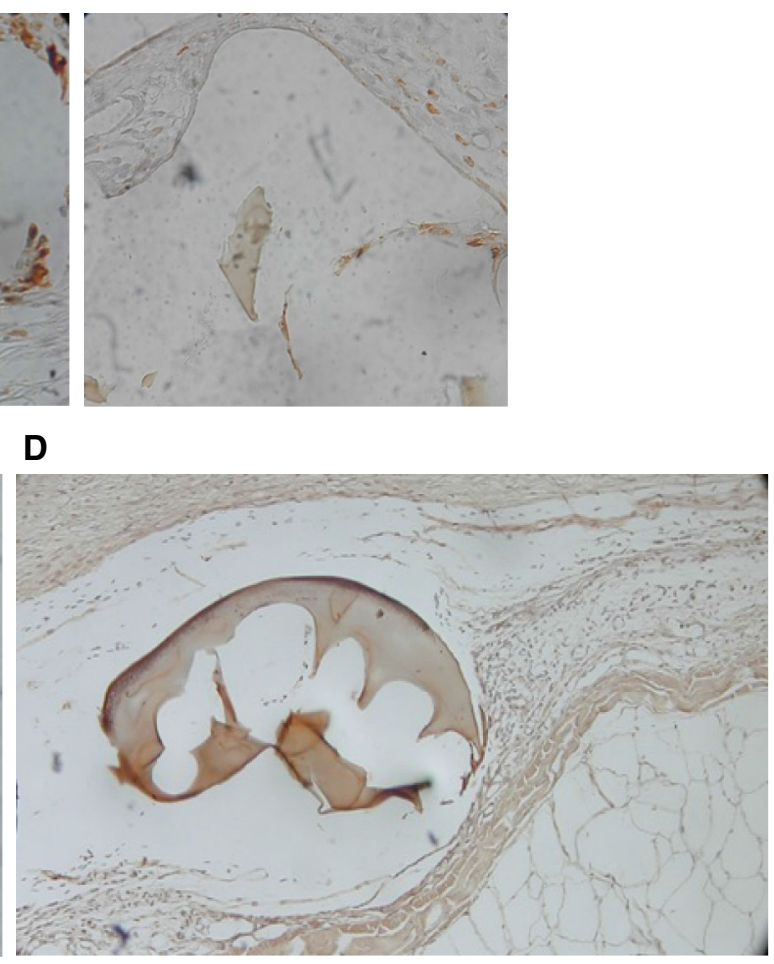

Coated PPM
Fig. 4 Macrophages and fibroblasts in coated and uncoated PPM samples. Macrophages were visualized with antibody to CD-68 in 12-week PPM samples. a Uncoated PPM. b Coated PPM. Fibroblasts were visualized with antibody to FSP-1 in 2-week PPM samples. c Uncoated PPM. d Coated PPM
Table 2 Apoptosis, oxidative stress and neovascularization in 2 and 12-week tissue

\begin{tabular}{llccc}
\hline & $\begin{array}{l}2 \text { weeks } \\
\text { Uncoated }\end{array}$ & $\begin{array}{l}2 \text { weeks } \\
\text { Coated }\end{array}$ & $\begin{array}{l}12 \text { weeks } \\
\text { Uncoated }\end{array}$ & $\begin{array}{l}12 \text { weeks } \\
\text { Coated }\end{array}$ \\
\hline TUNEL $^{+}$cells & $13.1 \pm 1.05$ & $1.5 \pm 0.2^{\bullet}$ & $11.0 \pm 1.1$ & $2.0 \pm 0.4^{\boldsymbol{\top}}$ \\
8-OHdG+ cells & $20.3 \pm 1.4$ & $6.7 \pm 1.2^{\bullet}$ & $31.9 \pm 2.3$ & $9.3 \pm 1.7^{\bullet}$ \\
Neovascularization \% & $9.01 \pm 1.47$ & $14.68 \pm 1.38^{* *}$ & $5.1 \pm .4$ & $9.6 \pm 0.5^{\bullet}$
\end{tabular}

Compared to Uncoated: $* * p<0.01 ;{ }^{\uparrow} p<0.001$ by proteins of higher affinity in a process known as the Vroman effect [14]. This results in attachment of proteins to the foreign implant, which leads to continued chronic FBR and subsequent fibrotic encapsulation and contraction of the implant. One of the unique features of the hydrogel coating used herein is shown in Fig. 1. The presence of both hydrophilic and hydrophobic sites on the hydrogel allows for a constant exchange of water molecules at the surface. Brownian motion ensures that water is constantly entering and leaving the polymer surface, preventing protein attachment which mitigates the Vroman effect. This was borne out in this study where it was shown that the number of foreign body giant cells found at 2 weeks was decreased by $95 \%$ and this persisted over 12 weeks. Fibrotic encapsulation of the coated PPM was also greatly reduced compared to uncoated PPM. Furthermore, when we examined the mesh/tissue interface for expression of Fibroblast specific protein-1, a marker for fibroblasts, we found a significant decrease in fibroblasts at both 2 and 12 weeks. Despite effects on the FBR, we do not anticipate effects of the coated mesh on wound healing in patients. Short-term mechanical strength is maintained due to the elastic and tissue-like bending modulus the mesh provides. Normal wound healing response provides the maximum healing response and viable scar tissue development to further support the surgical wound over time. Healthier scar tissue (well vascularized tissue) allows for tissue regeneration and immunologic resistance to infection. In the long term, the surgical wound is supported with the Mesh substrate, with intact vascular tissue providing 
Fig. 5 Apoptotic cells visualized with the TUNEL stain Apoptotic cells in 2-week PPM samples were visualized with the TUNEL stain. a 2week, uncoated PPM. b Area circled in $\mathbf{a}$ is enlarged in $\mathbf{b}$; individual nuclei are shown with arrows. c Coated PPM, showing almost no staining for apoptotic cells

\section{A}

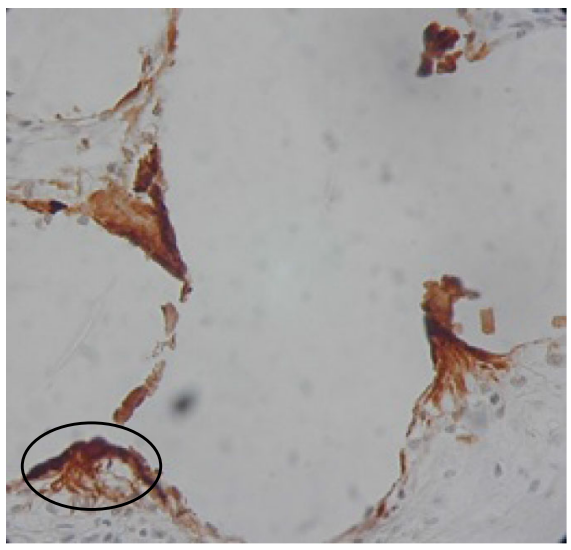

B

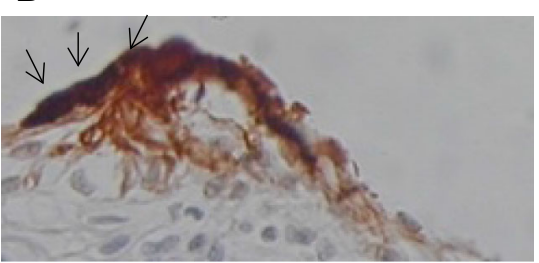

Uncoated PPM
C

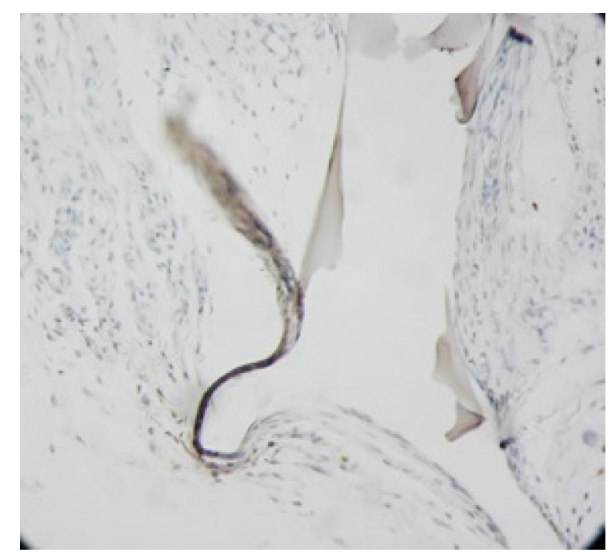

Coated PPM 


\section{A}

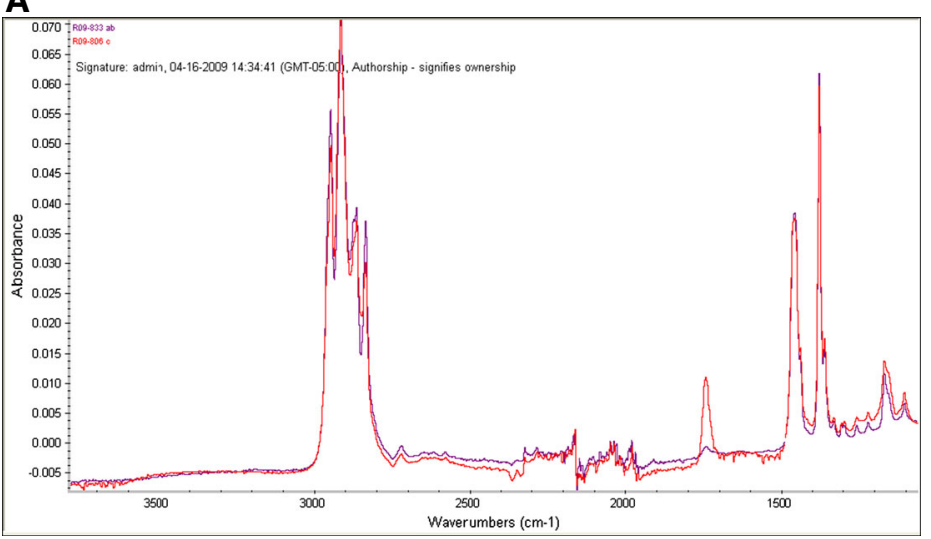

B

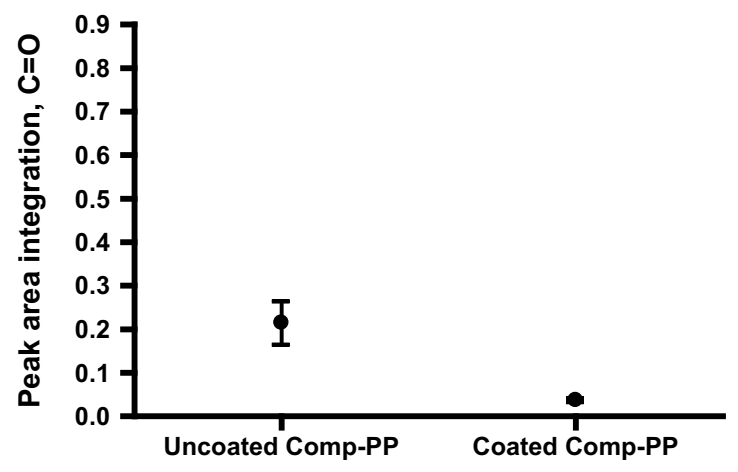

Fig. 7 Oxidative damage assessed with ATR-FTIR. Samples of PPM were removed from the rats and placed in sodium hypochlorite for $2 \mathrm{~h}$ (uncoated mesh) or $3 \mathrm{~h}$ (coated mesh). After cleaning and drying, the meshes were placed on the ATR crystal and subjected to ATR-FTIR. A Nicolet 6700 FT-IR spectrometer (Thermo Scientific, Waltham, MA, USA) was utilized to collect spectra with 32 scans and a resolution of $4 \mathrm{~cm}-1$ at ambient temperature. Three scans were performed per specimen. For the specimens, the presence of a carbonyl peak was analyzed via a peak area integration program in the OMNIC software package. The area of integration for the carbonyl peak, which peaks at $\sim 1740 \mathrm{~cm}^{-1}$, was from 1700 to $1775 \mathrm{~cm}^{-1}$. A A representative spectrum from ATR-FTIR experiments. Red line, uncoated PPM. Purple line, coated PPM. b Graphical representation of the results in shown in $\mathbf{a}$
A

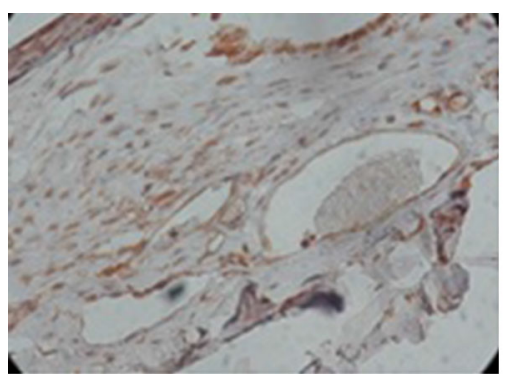

C

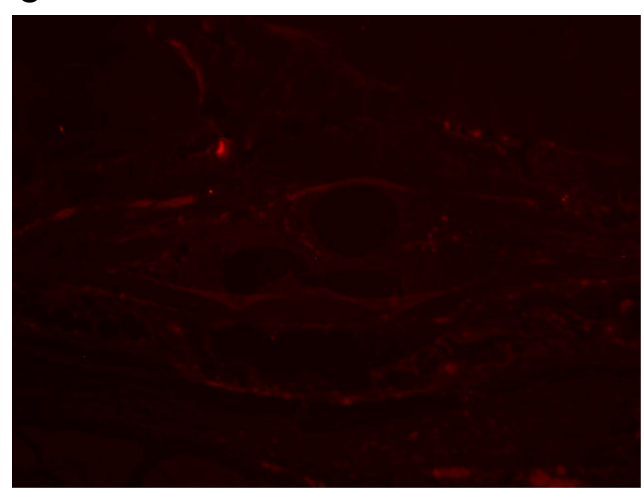

Uncoated PPM
B

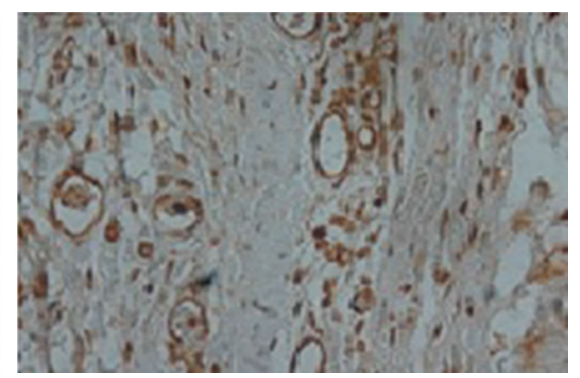

D

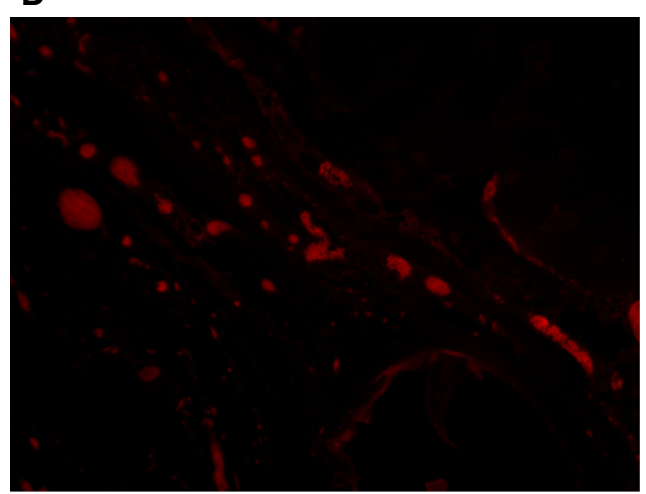

Coated PPM

Fig. 8 Neovascularization assessed in 2-week PPM samples. Neovascularization was assessed by staining with an antibody to CD-31, using either peroxidase $(\mathbf{a}, \mathbf{b})$ or fluorescence $(\mathbf{c}, \mathbf{d})$. Uncoated PPM is shown in a and $\mathbf{c}$, and coated PPM is shown in $\mathbf{b}$ and $\mathbf{d}$

replacement; it was shown that in that study that lack of neovascularization was associated with increased fibrosis [18].

It is thought that oxidation of mesh occurs in situ. In a 2007 study [19], mesh was taken from patients undergoing revision of their hernia repair. Indirect evidence of mesh oxidation was presented including scanning electron microscopy showing cracks, roughness and peeling of the mesh. In the present study, two different indicators of oxidative stress were examined. We demonstrated a 
Fig. 9 Matrix metalloprotease2 staining in 2-week PPM samples. Staining for MMP-2 is shown for representative sections of either. a Uncoated PPM or $\mathbf{b}$ coated PPM
A

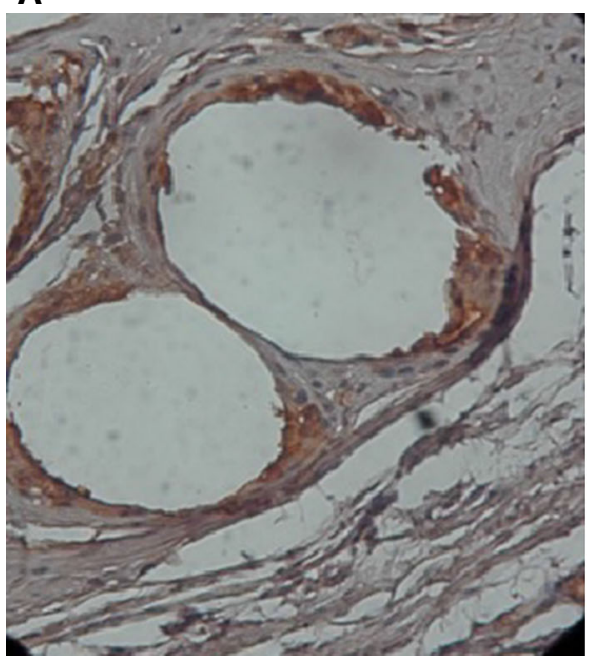

Uncoated PPM
B

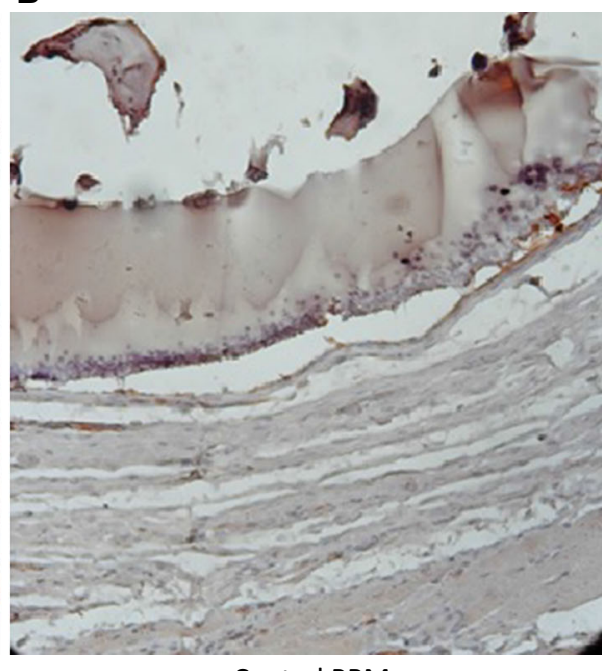

Coated PPM

significant decrease in $8-\mathrm{OHdG}$, a measure of DNA oxidation, in the coated PPM samples at both 2 and 12 weeks. We also used ATR-FTIR analysis [12], which detects the vibration characteristics of chemical functional groups in a mesh sample. Chemical bonds will stretch, contract and bend when infrared light interacts with the mesh sample. Utilizing ATR-FTIR, the specimens were analyzed to determine if the presence of functional groups such as $\mathrm{C}=\mathrm{O}$ were present on the surface. In this study, a significant decrease in these oxidative species was documented in the coated PPM compared to uncoated PPM. Thus, this study provides direct evidence of both oxidation of PPM in vivo, and its mitigation by the hydrogel coating.

It has also been shown that reactive oxygen and nitrogen (RON) species can activate MMP-2 in the heart [20]. Biosynthesis of peroxynitrite, an RON, led to acute mechanical dysfunction of myocardium followed by an increase in MMP-2 activity; this was mitigated by inhibitors of MMP-2. Infusion of RONS into the heart also caused dysfunction, which was mitigated by MMP-2 inhibitors. We showed a qualitative distinction between coated PPM and uncoated PPM in this study, with decreased expression of MMP-2 in the coated samples. Thus, this indirectly confirms a decreased oxidative stress response with coated PPM.

\section{Conclusion}

In summary, the results of the current study provide evidence that using this non-biodegradable hydrogel coating provides a PPM which when placed in rats for 2 and 12 weeks results in almost no fibrosis or foreign body response, limited oxidative stress and metalloprotease activity, and no evidence of infection. Clinical outcomes in hernia patients using the hydrogel coated mesh will need to be performed to confirm these important findings. Such studies could include monitoring surgical complications, deep wound infection, seroma formation, adhesions and the incidence of chronic pain, beginning at 3 months and continuing to 12 months. Long-term outcomes could include post-procedure mesh infections beyond 12 months to 5 years, and continued chronic pain syndrome (i.e. hard abdomen).

Acknowledgments Funding: Supported by The Frederick J. and Theresa Dow Wallace Fund of the New York Community Trust (DF, DP). This work was also supported by The Grateful Foundation and the Simon and Eve Colin Foundation. We thank SLB for excellent technical assistance in the FTIR experiments.

\section{Compliance with ethical standards}

Conflict of interest DP: declares conflict of interest directly related to the submitted work (founder and co-owner, stock ownership), DP also serves on the Scientific Board of the Cares Foundation.

JJS: declares no conflict of interest.

CMM: declares no conflict of interest.

JC: declares no conflict of interest.

JPT: declares no conflict of interest.

BJR: declares no conflict of interest.

DF: declares conflict of interest directly related to the submitted work (investment).

Open Access This article is distributed under the terms of the Creative Commons Attribution-NonCommercial 4.0 International License (http://creativecommons.org/licenses/by-nc/4.0/), which permits any noncommercial use, distribution, and reproduction in any medium, provided you give appropriate credit to the original author(s) and the source, provide a link to the Creative Commons license, and indicate if changes were made. 


\section{References}

1. Usher FC, Ochsner J, Tuttle LL Jr (1958) Use of Marlex mesh in the repair of incisional hernias. Am Surg 24:969-974

2. Klinge U (2008) Mesh for hernia repair. Br J Surg 95:539-540

3. Sanders D, Waydia S (2014) A systematic review of randomised control trials assessing mesh fixation in open inguinal hernia repair. Hernia 18:165-176

4. Harth KC, Rosen MJ (2009) Major complications associated with xenograft biologic mesh implantation in abdominal wall reconstruction. Surg Innov 16:324-329

5. Welty G, Klinge U, Klosterhalfen B, Kasperk R, Schumpelick V (2001) Functional impairment and complaints following incisional hernia repair with different polypropylene meshes. Hernia 5:142-147

6. Robinson T, Clarke J, Schoen J, Walsh M (2005) Major meshrelated complications following hernia repair. Surg Endosc Other Interv Tech 19:1556-1560

7. Bryers JD, Giachelli CM, Ratner BD (2012) Engineering biomaterials to integrate and heal: the biocompatibility paradigm shifts. Biotechnol Bioeng 109:1898-1911

8. Sadava EE, Krpata DM, Gao Y, Rosen MJ, Novitsky YW (2014) Wound healing process and mediators: implications for modulations for hernia repair and mesh integration. J Biomed Mater Res Part A 102:295-302

9. Rosen MJ (2009) Polyester-based mesh for ventral hernia repair: is it safe? Am J Surg 197:353-359

10. Miyajima A, Chen J, Lawrence C, Ledbetter S, Soslow RA, Stern J, Jha S, Pigato J, Lemer ML, Poppas DP, Vaughan ED, Felsen D (2000) Antibody to transforming growth factor-beta ameliorates tubular apoptosis in unilateral ureteral obstruction. Kidney Int 58:2301-2313

11. Mizuguchi Y, Chen J, Seshan SV, Poppas DP, Szeto HH, Felsen D (2008) A novel cell-permeable antioxidant peptide decreases renal tubular apoptosis and damage in unilateral ureteral obstruction. Am J Physiol Renal Physiol 295:F1545-F1555

12. Sarroukh R, Goormaghtigh E, Ruysschaert J, Raussens V (2013) ATR-FTIR: a "rejuvenated" tool to investigate amyloid proteins. Biochimica et Biophysica Acta (BBA)-Biomembranes 1828:2328-2338

13. Strutz F, Okada H, Lo C, Danoff T, Carone R, Tomaszewski J, Neilson E (1995) Identification and characterization of a fibroblast marker: FSP1. J Cell Biol 130:p393-p405

14. Vroman L (1962) Effect of adsorbed proteins on the wettability of hydrophilic and hydrophobic solids

15. Junge K, Binnebösel M, von Trotha KT, Rosch R, Klinge U, Neumann UP, Jansen PL (2012) Mesh biocompatibility: effects of cellular inflammation and tissue remodelling. Langenbeck's Arch Surg 397:255-270

16. Melman L, Jenkins E, Hamilton N, Bender L, Brodt M, Deeken C, Greco S, Frisella M, Matthews B (2011) Early biocompatibility of crosslinked and non-crosslinked biologic meshes in a porcine model of ventral hernia repair. Hernia 15:157-164

17. Patel ZS, Mikos AG (2004) Angiogenesis with biomaterial-based drug-and cell-delivery systems. J Biomater Sci Polym Ed 15:701-726

18. Menger M, Hammersen F, Messmer K (1992) In vivo assessment of neovascularization and incorporation of prosthetic vascular biografts. Thorac Cardiovasc Surg 40:19-25

19. Costello C, Bachman S, Ramshaw B, Grant S (2007) Materials characterization of explanted polypropylene hernia meshes. J Biomed Mater Res B Appl Biomater 83:44-49

20. Jacob-Ferreira AL, Schulz R (2013) Activation of intracellular matrix metalloproteinase- 2 by reactive oxygen-nitrogen species: consequences and therapeutic strategies in the heart. Arch Biochem Biophys 540:82-93 